\title{
Perfil dos Trabalhadores da Atenção Básica em Saúde no Município de São Paulo: região norte e central da cidade'
}

\section{Profile of the Primary Health Care Workers in the Municipality of São Paulo: north and central regions of the city}

\author{
Regina Maria Giffoni Marsiglia \\ Doutora em Ciência Política. Professora Adjunta da Faculdade de \\ Ciências Médicas da Santa Casa de São Paulo e Assistente Dou- \\ tora da Pontifícia Universidade Católica de São Paulo. Membro \\ da equipe do Observatório de Recursos Humanos em Saúde de \\ São Paulo. \\ Endereço: Cealag - Rua Cesário Mota Jr., 6I, 5 andar, CEP 01221-000, \\ Vila Buarque, São Paulo, SP, Brasil. \\ E-mail: regina.marsiglia $₫ \mathrm{fcmscsp}$.edu.br \\ I Apoio financeiro: OPAS/MS - Carta Acordo: BR/LOA/0600029.001/ \\ 002/003/004, 2008/2009.
}

\section{Resumo}

Pesquisa do Observatório de Recursos Humanos em Saúde de São Paulo em 2009, com 87 UBS na região norte e centro da cidade. Objetivo: levantar o perfil dos trabalhadores das UBS, organizadas sob: Programas de Saúde, Saúde da Família e Mistas. Metodologia: sexo, idade, naturalidade, escolaridade, formação, função e tempo de trabalho na UBS de 4.968 trabalhadores, e entrevistas com amostra de 621 trabalhadores, através do Sistema de Entrevista Telefônica Assistida por Computador (ETAC). Resultados: 80,7\% dos entrevistados eram mulheres, mas só 56,4\% entre os médicos; 50,5\% entre 21 e 40 anos de idade e 49,1\% entre 41 e 60 anos; $61,4 \%$ naturais do município ; apresentaram escolaridade superior ao exigido para as funções exercidas; no nível superior, 13,9 \% eram médicos; no médio, 21,3\% eram auxiliares de enfermagem e, no operacional, 20,7\% eram ACS; 40,1\% trabalhavam na UBS há mais de 5 anos, 12,9\% há mais de 10 anos e 6,3\% há mais de 20 anos; $64,7 \%$ trabalhavam só na UBS, 46,7\% tiveram experiência na área hospitalar; 92,3\% dos que trabalhavam nas UBS de Programas de Saúde eram funcionários públicos estatutários e $77 \%$ dos das UBS de Saúde da família contratados via CLT, pelas instituições parceiras da SMS-SP. Conclusões: predominância de trabalhadores do sexo feminino; processos de consolidação e de renovação do contingente de trabalhadores, maioria natural do município de São Paulo, escolaridade superior à exigida para a função; predominância de médicos, auxiliares de enfermagem e agentes comunitários de saúde; processo de terceirização nas relações de trabalho.

Palavras-chave: Atenção Primária à Saúde; Modelos de Organização; Recursos Humanos. 


\section{Abstract}

Research conducted by São Paulo's Observatory of Human Resources in Health in 2009. It investigated 87 unidades básicas de saúde (UBS - primary care units) located in the north and central regions of the city of São Paulo. Objective: to survey the profile of the UBS workers. The UBS were organized under different models: Health Programs, Family Health and mixed. Methodology: information about the universe of 4,968 workers was surveyed in the SMSSP (Municipal Health Department of São Paulo) and interviews were conducted with a sample of 621 workers through the ETAC System (ComputerAssisted Telephone Interview). Results: $80.7 \%$ of the interviewees were women, but only $56.4 \%$ among the physicians; $50.5 \%$ were aged $21-40$ years and $49.1 \%$ were aged $41-60$ years; $61.4 \%$ were born in the city of São Paulo; a great deal of them presented a higher level of schooling than what was required to perform the functions; in the superior level, $13.9 \%$ were physicians; in the middle level, 21.3\% were nursing assistants; and in the operational level, 20.7\% were ACS (Community Health Agents); 40.1\% had been working at the UBS for more than five years, $12.9 \%$ for more than 10 years and $6.3 \%$ for more than 20 years; $64.7 \%$ worked only at the UBS, $46.7 \%$ had had a previous experience in the hospital area; 92.3\% of those who worked at the Health Programs UBS were statutory civil servants, and $77 \%$ of those of the Family Health UBS were hired through Brazil's Labor Laws, by the partner institutions of the SMSSP. Conclusions: predominance of female workers; process of consolidation and renewal of the quota of workers; the majority was born in the city of São Paulo and had a level of schooling that was higher than what was required for the function; predominance of doctors, nursing assistants and community health agents; outsourced contracts of employment.

Keywords: Primary Health Care; Organization Models; Human Resources.

\section{Introdução}

Nesta pesquisa sobre os Recursos Humanos na Atenção Básica em Saúde na cidade de São Paulo, consideraram-se os diferentes modelos de organização que esses serviços apresentavam em 2009, resultado do processo histórico que se desenvolve há mais de quatro décadas na cidade, fruto das políticas desenvolvidas pela Secretaria Municipal e Estadual de Saúde de São Paulo. As Unidades Básicas de Saúde (UBS) eram organizadas sob dois modelos: os Programas de Saúde (Adulto, Bucal, Criança, Mental e Mulher, Hanseníase e Tuberculose), implantados durante a década de 1970 e que permaneceram como modelo único até meados dos anos 1990 (Nemes, 1993; Luppi e col., 2008), e o modelo proposto pelo Ministério da Saúde para a Atenção Básica, através da Estratégia de Saúde da Família (Brasil, 1997).

O modelo de Estratégia de Saúde da Família começou a ser implantado na cidade de São Paulo sob denominação de Qualidade Integral em Saúde (Qualis) a partir de 1996 pela Secretaria de Estado da Saúde, através de convênios com quatro instituições parceiras: Casa de Saúde Santa Marcelina, Fundação Zerbini, Associação Congregação de Santa Catarina e Universidade de Santo Amaro (Unisa) -, pois, naquele momento, a administração municipal havia implantado o Plano de Assistência à Saúde (PAS), baseado em cooperativas vinculadas ao setor privado de assistência (Jatene e col., 200o; Silva e Dalmaso, 2002) ${ }^{2}$.

A Estratégia da Saúde da Família só passou a ser desenvolvida sob responsabilidade da administração municipal a partir de 2001, quando o PAS foi desativado. A partir de então, as 220 equipes do Qualis foram transferidas para a gestão municipal, e novas equipes de Saúde da Família foram implantadas, preferencialmente nos distritos administrativos de maior exclusão social, mantendo-se o sistema de parcerias e ampliando-se para 12 o número de instituições parceiras da Secretaria Municipal da Saúde de São Paulo (SMS-SP) (Cohn e col., 2005).

Neste contexto, na maioria dos distritos, não foram implantadas novas UBS, mas aproveitou-se

2 OBSERVATÓRIO DE RH EM SAÚDE DE SÃO PAULO: Recursos Humanos em Atenção Básica à Saúde e o Programa de Saúde da Família: Biênios 2006/2007; 2008/2009. São Paulo: Observatório de RH em São Paulo, [s.d.]. Disponível em: <http://www.observarhsp.org.br/ Projetos/rh.html>. Acesso em: 20 jun. 2010. 
a estrutura física das UBS municipais já existentes ou das estaduais que haviam sido municipalizadas. Assim, parte das UBS organizadas sob o modelo dos Programas de Saúde passaram a receber também equipes de Saúde da Família, e os dois modelos passaram a conviver no mesmo espaço físico - UBS Mistas -, o que tem gerado alguns conflitos entre os trabalhadores, profissionais e usuários dos dois modelos de organização (Marsiglia e Carneiro Junior, 2009).

A partir de 2005, a SMS-SP foi dividida em cinco Coordenadorias Regionais de Saúde e estas, por sua vez, em 25 Supervisões Técnicas de Saúde. Ao final de 2009, quando esta pesquisa terminou, o município de São Paulo contava com 420 UBS, dentre as quais $37,8 \%$ eram organizadas exclusivamente sob o modelo de Saúde da Família. Foram implantadas 1.052 equipes, através de convênios estabelecidos da SMSSP com 12 instituições parceiras. A distribuição das equipes de Saúde da Família pelas Coordenadorias Regionais de Saúde era a apresentada na tabela 1.

Tabela I - Distribuição do número e proporção (\%) das equipes de Saúde da Família por Coordenadoria Regional de Saúde. Município de São Paulo, 2009

\begin{tabular}{lcc} 
Coordenadoria & N & $\%$ \\
Coordenadoria Regional de Saúde Sudeste & 169 & 16,1 \\
Coordenadoria Regional de Saúde Centro-Oeste & 70 & 6,7 \\
Coordenadoria Regional de Saúde Norte & 164 & 15,6 \\
Coordenadoria Regional de Saúde Sul & 427 & 40,6 \\
Coordenadoria Regional de Saúde Leste & 222 & 21,1 \\
\hline Total & 1.052 & 100,0 \\
\hline
\end{tabular}

Fonte: Coordenadoria de Atenção Básica de Saúde da Secretaria Municipal de Saúde de São Paulo. São Paulo, 2009.

O objetivo principal da pesquisa foi levantar e comparar o perfil dos trabalhadores que estavam atuando na Atenção Básica na cidade de São Paulo em 2008-2009, nas UBS organizadas exclusivamente sob um dos modelos: o dos Programas em Saúde ou o da Saúde da Família, bem como daqueles que trabalhavam em UBS Mistas, em que coexistiam os dois modelos.

\section{Metodologia}

Campo de pesquisa: a escolha das UBS da Coordenadoria Regional de Saúde Norte e de parte das UBS da Coordenadoria Regional de Saúde Centro-Oeste (Supervisão Técnica de Saúde Sé) para este estudo se justifica por várias razões. Primeiramente, porque as relações entre a Faculdade de Ciências Médicas da Santa Casa de São Paulo (FCMSCSP) e a Irmandade da Santa Casa de Misericórdia de São Paulo (ISCMSP) com as Secretarias Municipal e Estadual de Saúde de São Paulo são muito antigas. Em 1966, essas instituições formalizaram entre si um convênio para a implantação do Centro de Saúde Experimental da Barra Funda e Bom Retiro (atualmente Centro de Saúde Escola Barra Funda Dr. Alexandre Vranjac), do Programa de Integração DocenteAssistencial (PIDA); de 1982 a 1994, a ISCMSP foi uma das parceiras da SMS-SP para a implantação de equipes de Saúde da Família desde 2001 e de Núcleos de Apoio às Equipes de Saúde da Família (NASF) nessas áreas desde 2009; vêm participando da articulação com os Ministérios da Saúde e da Educação para o desenvolvimento dos Programas de Reorientação da Formação de Graduação em Saúde para a Atenção Básica (Pró-Saúde) e do Programa de Educação pelo Trabalho (PET-Saúde) desde 2005 (Brasil, 2005, 2008; Marsiglia e col., 2010). Os compromissos e parcerias da assumidas pela FCMSCSP e ISCMSP com o poder público, nos últimos 40 anos, apontam para projetos e programas direcionados à região norte e à área central da cidade de São Paulo, tendo em vista a regionalização da atenção à saúde, a educação permanente dos profissionais da rede pública de saúde, a formação de técnicos (Profae) e profissionais de nível superior, o desenvolvimento de pesquisas e atividades de extensão.

É preciso considerar também que na região norte e na área central da cidade de São Paulo, o desenvolvimento da Atenção Básica é um processo antigo e de grande extensão. A implantação de UBS organizadas sob o modelo dos Programas de Saúde começou nos anos 1970, quando das reformas da Secretaria de Estado da Saúde de São Paulo (SES-SP), durante a gestão do Prof. Valter Leser e da SMS-SP (na época, ainda Secretaria de Higiene e Saúde), durante a gestão do Prof. Cornélio Pedroso Rosenburg (Marsiglia, 1993). 
Posteriormente, a partir de 1996, parte das UBS localizadas na região norte também participou do projeto Qualis (1996-2001) e, a partir de 2001, outras foram reorganizadas sob modelo de Saúde da Família, sendo todas as equipes contratadas por instituições parceiras da SMS-SP. A população atendida pelas UBS da região norte e da área central da cidade é bastante diversificada e heterogênea do ponto de vista demográfico, social e de acesso aos serviços de saúde, o que se reflete nos indicadores de saúde, que, para alguns segmentos da população, estão bem próximos da média do município, mas, para outros segmentos, podem estar acima ou abaixo dessa média.

$\mathrm{O}$ projeto foi apresentado às Coordenadorias Regionais de Saúde Norte e Centro Oeste, na Supervisão Técnica de Saúde Sé (STS Sé), e discutido com os gerentes das UBS que seriam pesquisadas e com os responsáveis pelas instituições parceiras da SMS-SP, para a contratação das Equipes de Saúde da Família para as UBS dessas regiões, solicitando-se sua colaboração, o fornecimento das informações e a autorização para a aplicação dos instrumentos de coleta.

\section{Fontes de dados e instrumento de pesquisa:}

- Informações obtidas junto às Coordenadorias de Recursos Humanos e de Atenção Básica/PSF da SMS-SP, da Coordenadoria Regional de Saúde Norte e da Supervisão Técnica de Saúde Sé e das instituições parceiras da SMS-SP para a operacionalização do PSF nessas áreas, considerando-se o universo de trabalhadores das UBS existentes nessas regiões: sexo, idade, naturalidade, escolaridade, formação, função atual, tempo de trabalho na unidade.

- Aplicação de formulário por telefone através do sistema de Entrevista Telefônica Assistida por Computador (ETAC) a uma amostra de trabalhadores das UBS dessas regiões, uma média de 6 a 10 entrevistados por unidade, incluindo gerentes, médicos, enfermeiros, outros profissionais de nível superior, auxiliares de enfermagem, agentes comunitários de saúde e funcionários administrativos. Nessas situações, o Termo de Consentimento Livre e Esclarecido (TCLE) foi substituído pelo consentimento verbal gravado. A previsão inicial era de que 629 pessoas fossem entrevistadas, mas como em algumas UBS o quadro de pessoal não estava completo e em outras houve recusas, foram entrevistadas 621 pessoas.

\section{Resultados}

\section{Universo de trabalhadores da Atenção Básica nas UBS da Coordenadoria Regional de Saúde Norte e Supervisão Técnica de Saúde Sé}

\section{Total de trabalhadores por UBS}

O universo constituído por 4.968 trabalhadores, atuando nas 87 UBS das regiões pesquisadas, 81 unidades localizadas na Coordenadoria Regional de Saúde Norte e seis na Supervisão Técnica de Saúde Sé, foram assim distribuídos (gráfico 1).

\section{Gráfico I - Distribuição das UBS e Trabalhadores por Supervisões Técnicas de Saúde, região norte e Sé. SMS-SP, 2009}

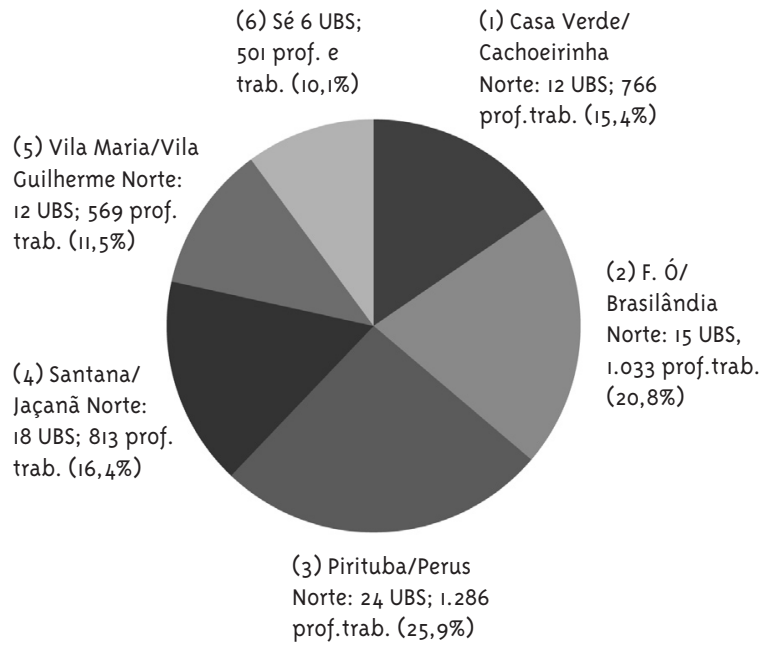

Fonte: Coordenadorias de RH da SMS-SP, da Região Norte e da STS Sé. São Paulo, 2009.

A STS de Pirituba/Perus concentrava o maior número de UBS e o maior percentual de trabalhadores (25,9\%), seguida da Freguesia do Ó/Brasilândia (20,8\%). A STS Sé contava com apenas seis UBS e o menor número de trabalhadores (10,1\%). No entanto, se dividirmos o total de trabalhadores pelo número de UBS em cada STS, verificamos, que as UBS da STS Sé, contavam com um contingente maior de recursos humanos por UBS: em média, elas tinham 83,5 trabalhadores; as UBS das STS F.Ó/Brasilândia e STS 
Casa Verde/Cachoerinha, respectivamente tinham 68,9 e 63,8; as UBS da STS Perus/Pirituba, 53,6; as UBS da STS V. Maria/V. Guilherme e as UBS da STS Santana/Jaçanã, respectivamente, 47,4 e 45,2.

Considerando a distribuição das UBS e dos trabalhadores por modelo de organização, observou-se que entre as UBS pesquisadas $49,4 \%$ eram UBS organizadas exclusivamente sob o modelo de Programas de Saúde e absorviam 40,6\% dos trabalhadores em Atenção Básica dessas regiões, 26,4\% estavam organizadas exclusivamente sob o modelo de Saúde da Família, e contavam com 28,5\% desses trabalhadores e, 24,1\% eram UBS Mistas, que absorviam 30,8\% dos trabalhadores.

\section{Perfil dos trabalhadores}

Dos trabalhadores, $80,7 \%$ eram do sexo feminino, $50,5 \%$ tinham entre 21 e 40 anos de idade e $49,1 \%$ entre 41 e 60 anos; 72,6\% eram naturais da região Sudeste do país, 9,5\% da região Nordeste, o,4\% da região Centro-Oeste e $1 \%$ de outros países.

\section{Formação e capacitação}

Como nos bancos de dados consultados não constavam informações precisas sobre a escolaridade e a formação para o universo de trabalhadores, considerou-se a distribuição dos recursos humanos segundo o nível de estudo exigido para o preenchimento dos cargos, conforme listagem da Coordenadoria de RH da SMS-SP.

As exigências eram as seguintes: superior completo para cargos de Assistente Social, Chefe de Seção, Cirurgião Dentista, Coordenador de Unidade Básica, Enfermeiro, Farmacêutico, Fonoaudiólogo, Médico, Nutricionista, Psicólogo, Terapeuta Ocupacional, entre outros de nível superior. Escolaridade de nível médio completo para: Assistente Administrativo, Oficial Administrativo, Auxiliar de Consultório Dentário, Técnico de Farmácia, Técnico de Saúde - Higiene Dental, Técnico de Saúde - Prótese Dentária, Encarregado de Equipe, entre outros, encontrando-se no mesmo nível trabalhadores auxiliares e ocupações de nível técnico. Escolaridade de nível fundamental completo para Cargos de Nível Operacional: Agente Comunitário de Saúde (ACS), Atendente de Enfermagem, Agente de Apoio, Auxiliar de Serviços Gerais, Motorista, Servente, Vigia, entre outros; alguns cargos são equivalentes ao cargo de Agente de Apoio.

De acordo com essas exigências, verificou-se que 30,8\% ocupavam cargos que exigem nível superior completo de escolaridade, $38,6 \%$ cargos que exigem nível médio de escolaridade, e, 30,5\%, cargos de nível fundamental de escolaridade.

\section{Profissões e ocupações}

Considerando as profissões e ocupações mais frequentes nessas UBS, constatou-se que entre as de nível superior, os profissionais médicos eram os mais frequentes (13,9\%), seguidos de enfermeiros (6\%), e cirurgiões-dentistas (5\%). Um segundo grupo bem menor era constituído por psicólogos $(1,3 \%)$ e assistentes sociais $(1,2 \%)$, podendo indicar que a maioria das UBS contava com pelo menos um desses profissionais incorporados na Atenção Básica em São Paulo desde as propostas dos anos 1970. Um terceiro grupo, encontrado em aproximadamente $23 \%$ das UBS, era composto de fonoaudiólogos e educadores em saúde pública, representando o, $4 \%$ dos profissionais dessas UBS, e os menos frequentes, encontrados em menos de $10 \%$ das UBS, foram fisioterapeutas, terapeutas ocupacionais e nutricionistas, cada um deles representando o,2\% dos recursos humanos nas UBS pesquisadas. Havia ainda apenas dois farmacêuticos nas 87 UBS pesquisadas.

Entre as ocupações que exigiam nível médio de escolaridade, predominaram os "auxiliares", que constituíam 94,3\% do contingente de nível médio, enquanto apenas 5,7\% eram trabalhadores que ocupavam cargos de nível técnico. Entre os auxiliares, $21,3 \%$ eram auxiliares de enfermagem, $11,1 \%$ agentes de gestão de políticas públicas, 2,6\% auxiliares de consultório dentário, e 1,4\% pessoal administrativo auxiliar.

As ocupações que exigiam nível técnico estavam assim constituídas: oficiais administrativos (o,9\%), técnicos em farmácia e higiene dental (cada um representando o, $3 \%$ do pessoal), e técnicos em radiologia, representando apenas o,1\% do contingente.

No seguimento dos trabalhadores de nível operacional, os mais frequentes foram os agentes comunitários de saúde (20,7\%) e, bem menos frequentes, os agentes de apoio (3,6\%), auxiliares de serviços gerais $(2,3 \%)$, atendentes $(2 \%)$, atendentes de enfermagem (2\%), visitadores sanitários e vigias (cada um com $\mathrm{o}, 4 \%$ ), serventes (o,2\%) e motoristas (o,1\%). 
Contingente de trabalhadores, escolaridade e modelo de organização das UBS

As UBS organizadas sob o modelo dos Programas de Saúde concentravam 40,6\% dos recursos humanos, com uma média de 46,9 pessoas por UBS assim aproximadamente distribuídas: 21 trabalhadores de nível médio de escolaridade (auxiliares e técnicos), 18 de nível superior e oito de nível operacional. Nas UBS organizadas sob o modelo da Saúde da Família, encontrava-se $28,5 \%$ do universo pesquisado, mas cada UBS contava, em média, com um maior número de trabalhadores, isto é, havia cerca de 61,6 trabalhadores por UBS, destacando-se, nesses casos, a presença significativa de trabalhadores operacionais, em média 29 por UBS, provavelmente em virtude da incorporação dos ACS nas equipes. Nessas UBS, foram encontrados cerca de 20 trabalhadores auxiliares e técnicos e o menor contingente de profissionais de nível universitário, aproximadamente 13 por UBS. As UBS Mistas absorviam 30,8\% do universo de trabalhadores e profissionais, com média de 72,9 pessoas por UBS: em média 27 trabalhadores de nível auxiliar e técnico, 25 de nível operacional, também reforçados pelo contingente dos ACS das equipes de Saúde da Família, e 21 profissionais de nível universitário. Nas UBS do modelo de Saúde da Família, 91,5\% dos trabalhadores de Nível Operacional eram Agentes Comunitários de Saúde, e nas unidades mistas, os ACS também já constituíam 8o,2\% dos trabalhadores de nível operacional, enquanto nas UBS do modelo Programas de Saúde, o maior percentual de trabalhadores de nível operacional era constituído por agentes de apoio (29,8\%). Nos cargos que exigiam nível médio de escolaridade, os auxiliares de enfermagem eram maioria em todas as UBS, independentemente do modelo de organização. Representavam 52,4\% dos trabalhadores de nível médio no conjunto das UBS do modelo de Programas de Saúde, 6o\% desses trabalhadores no conjunto das UBS Mistas e $54,7 \%$ dos trabalhadores desse nível no conjunto das UBS da Saúde da Família. Foi possível observar ainda, que, embora no conjunto de profissionais de nível superior, os médicos fossem maioria, quando essa informação foi analisada em relação ao modelo de organização das UBS, nas UBS do modelo de Saúde da Família, o número total de enfermeiros ficou muito próximo do número total de médicos: uma média de 4,6 enfermeiros e 4,5 médicos por UBS, provavelmente pela composição das equipes de Saúde da família. No conjunto das UBS do modelo Programa de Saúde, foi encontrada média de 8,7 médicos por UBS, e no conjunto das UBS Mistas, média de 10,1 médicos por UBS, somando-se os médicos que atuavam nos diversos Programas de Saúde e nas equipes de Saúde da Família.

Amostra de trabalhadores da Atenção Básica da Coordenadoria Regional de Saúde Norte e Supervisão Técnica de Saúde Sé entrevistados pelo Sistema ETAC

\section{Distribuição dos entrevistados por Supervisão Téc- nica de Saúde e modelo de organização das UBS}

Tabela 2 - Distribuição do número e proporção (\%) de elementos da amostra de entrevistados ETAC por Supervisões Técnicas de Saúde. SMS-SP, 2009

\begin{tabular}{lcc} 
Total Banco Geral & 4.968 & $100,0 \%$ \\
Total de Entrevistas & 621 & $12,5 \%$ \\
\hline STS Casa Verde/Cachoeirinha & 87 & $14,0 \%$ \\
STS Freguesia do Ó/Brasilândia & 107 & $17,2 \%$ \\
STS Santana/Jaçanã & 129 & $20,8 \%$ \\
STS Pirituba/Perus & 166 & $26,7 \%$ \\
STS Vila Maria/Vila Guilherme & 79 & $12,7 \%$ \\
STS Sé & 53 & $8,5 \%$ \\
\hline
\end{tabular}

Fonte: Coordenadoria de RH da SMS-SP, Região Norte e STS Sé. Sé da Região CentroOeste da Cidade de São Paulo. São Paulo, 2009.

Considerando o modelo de organização das UBS em que estavam inseridos os trabalhadores entrevistados, a seguinte distribuição foi encontrada: $41,2 \%$ deles trabalhavam em UBS do Modelo de Programas de Saúde, 33,4\% em UBS Mistas e 25,4\% em UBS do modelo de Saúde da Família.

\section{Profissões e ocupações dos entrevistados}

Do ponto de vista de suas categorias profissionais ou ocupacionais, os entrevistados apresentaram a seguinte distribuição: 7,1\% eram ACS, 17,6\% auxiliares de enfermagem, $14 \%$ pertenciam à área administrativa, $14 \%$ eram coordenadores ou gerentes 
das unidades, $17,4 \%$ médicos, $17,4 \%$ enfermeiros, e $12,6 \%$ pertenciam à equipe ampliada (pessoal de nível universitário, não médico ou enfermeiro), dentre os quais: 13 eram assistentes sociais, 44 cirurgiões-dentistas, 3 educadores em saúde pública, 1 farmacêutico, 1 fisioterapeuta, 1 fonoaudiólogo, 3 nutricionistas, 11 psicólogos e 1 terapeuta- ocupacional.

\section{Perfil dos entrevistados}

Houve predominância de entrevistados do sexo feminino (79,4\%), independentemente do modelo de organização da UBS, embora essa tendência se apresentasse um pouco mais acentuada nas UBS organizadas sob o modelo de Programas de Saúde: nessas UBS, $80,1 \%$ eram mulheres, o mesmo ocorrendo com $79,3 \%$ dos recursos humanos das UBS Mistas e 78,3\% das UBS de Saúde da Família. Em todas as categorias profissionais, o sexo feminino foi predominante, especialmente entre os enfermeiros (90,3\%). A tendência à feminização entre os médicos, mesmo existente, mostrou-se menos acentuada: $56,4 \%$ dos médicos eram do sexo feminino. Do ponto de vista da faixa etária, na amostra de entrevistados, encontrou-se um contingente maior de trabalhadores entre 40 e 59 anos $(67,6 \%)$, enquanto no universo pesquisado, 49,1\% tinham entre 41 e 60 anos de idade. 0 envelhecimento da força de trabalho foi uma tendência encontrada em todas as UBS, independentemente do modelo de organização, mas esse processo foi um pouco mais acentuado nas UBS do modelo de Programas de Saúde, dado a antiguidade de implantação dessas UBS, nos anos 1970. Foi menor entre os que trabalhavam nas UBS de Saúde da Família, implantadas mais recentemente, durante os anos 1990 através do Projeto Qualis pela SES-SP, e a partir de 2001, pela SMS na cidade de São Paulo. Apenas entre os entrevistados das UBS de Saúde da Família encontraram-se trabalhadores com menos de 20 anos de idade.

Dentre os entrevistados, 55,6\% eram casados, $51 \%$ tinham um ou dois filhos e $18,8 \%$, três ou quatro filhos; $61,4 \%$ nasceram no município de São Paulo, $18,7 \%$ no interior do Estado de São Paulo, 18,7 \% eram de outros estados do país e 1,3\% de outros países.

Considerando-se a distribuição por modelo de organização das UBS, constatou-se que dentre os entrevistados nas UBS de Programas de Saúde, 66\% eram naturais do município de São Paulo, o mesmo ocorrendo com $63,1 \%$ dos entrevistados nas UBS de Saúde da Família, e com 54,3\% dos entrevistados nas UBS Mistas. Os nascidos no interior do estado de São Paulo constituíam 16,4\% dos entrevistados que trabalhavam nas UBS do modelo de Programas de Saúde, 19,7\% dos entrevistados das UBS de Saúde da Família, enquanto nas UBS Mistas, o segundo maior contingente de entrevistados (24\%) era natural de outros estados do país.

\section{Formação e capacitação}

Quanto à escolaridade, 70,2\% dos entrevistados iniciaram cursos de nível superior e destes $64 \%$ os completaram, o que aponta um alto grau de escolaridade, muitas vezes acima do exigido para o exercício dos cargos que ocupavam especialmente nos de nível médio e operacional; 52,8\% dos entrevistados informaram ter concluído Cursos de Especialização, em um total de 557 cursos, porque muitos participaram de mais de um curso de Especialização: 30,2\% terminaram um curso de Especialização; 44,2\% fizeram dois cursos, $18 \%$ fizeram três cursos, $7,3 \%$ fizeram quatro cursos. Esses dados reforçam a ideia de uma força de trabalho qualificada e que continua sua formação depois de inserida na Atenção Básica, em geral incentivada pela administração pública de saúde. Observando-se a distribuição dos profissionais com Curso de Especialização por modelo de organização da UBS, constatou-se seguinte situação: tinham Curso de Especialização 58,2\% dos que trabalhavam nas UBS do modelo de Programas de Saúde, $51,9 \%$ dos que trabalhavam nas UBS Mistas e 45,2\% dos que trabalhavam nas UBS de Saúde da Família. Analisando-se os 557 Cursos de Especialização frequentados pelos entrevistados, observa-se uma grande variação. Os mais citados foram: Saúde Pública (Medicina Preventiva e Saúde Coletiva), por 25,05\%; Pediatria, Puericultura, Neonatologia e Hebiatria, por 11,7\%; Clínica Médica, Cardiologia, Pneumologia, Endocrinologia e Geriatria, por 9,2\%; Ginecologia e Obstetrícia, por 7,2\%; Saúde da Família e Medicina de Família, por 6,3\%; GERUS, por 5,9\%; Pedagogia e Educação em Saúde, por 5,6\%; Administração Hospitalar e Gestão Pública, por 
4,8\% e Saúde Mental, por 4,7\%.

Entre os entrevistados, outros $32 \%$ informaram ter terminado cursos de nível técnico, sendo o mais citado o de Técnico de Enfermagem, seguido de cursos técnicos da área de administração, como Contabilidade e outros de Informática, embora com pouca frequência. Relacionando a realização de Cursos Técnicos com a inserção dos entrevistados nas UBS, segundo o modelo de organização dominante, constatou-se que 33,3\% dos entrevistados informaram trabalhar em UBS do modelo de Programas de Saúde, 36,7\% em UBS Mistas e 30,1\% em UBS de Saúde da Família. Quanto a treinamentos recebidos para trabalhar na Atenção Básica, 68,3\% dos entrevistados responderam afirmativamente, sendo 39,5\% trabalhavam nas UBS de Programas de Saúde, 25,3\% trabalhavam em UBS de Saúde da Família e 3,5\%dos trabalhavam nas UBS Mistas. Chama atenção, no entanto, que 31,4\% dos entrevistados informaram não ter recebido nenhuma capacitação para trabalhar na Atenção Básica. As capacitações mais citados pelos entrevistados foram: $16,7 \%$ para os Programas de Saúde da Criança, da Mulher, do Adulto, Mental e Bucal; 12,7\% informaram ter frequentado cursos dos GERUS, 8,4\% cursos sobre imunização, esterilização, curativos; $6 \%$ cursos sobre TB, hanseníase, dengue; 4,8\% cursos de Saúde Pública e sobre Comunidade e 4,4\% sobre recepção, acolhimento, cartão SUS e outras rotinas. Cursos para o trabalho em Saúde da Família, Momentos I e II somados, foram citados por apenas $4 \%$ dos entrevistados. Chama atenção o fato de $28,7 \%$ dos que afirmaram ter recebido capacitação para trabalhar na Atenção Básica não terem informado que curso tinham realizado, porque não se lembravam mais ou porque haviam realizado vários cursos e não aceitaram elencá-los. Entre os entrevistados que estavam trabalhando nas Equipes de Saúde da Família, 45 haviam trabalhado no Programa Qualis na segunda metade dos anos 1990 e foram incorporados pela SMS-SP com a municipalização dos serviços em 2001: entre estes, $82,2 \%$ afirmaram ter realizado cursos/treinamentos para desenvolver seu trabalho, enquanto $17,8 \%$ não tiveram nenhum treinamento para desenvolver suas atividades.

\section{Tempo de atuação na unidade atual}

Observou-se que 12,9\% dos entrevistados trabalhavam há mais de 10 anos na mesma UBS, e 6,3\% há mais de 20 anos, o que indica a existência de um contingente de trabalhadores estáveis e antigos; 23,7\% trabalhavam na UBS há apenas 1 ou 2 anos, e outros $14,2 \%$ há 3 ou 4 anos. Somando-se esses dois últimos contingentes, pode-se afirmar que 37,9\% trabalhavam na mesma UBS há menos de 5 anos, e 20,9\% há 5 ou 6 anos, indicando que um processo de renovação e incorporação de novos trabalhadores na Atenção Básica nas áreas pesquisadas vem se desenvolvendo. Quando se relaciona o tempo de trabalho dos entrevistados na mesma UBS com o modelo de organização, constata-se que, proporcionalmente, havia trabalhadores com mais anos de casa nas UBS do modelo de Programas em Saúde, o que era de se esperar, dada a antiguidade da implantação desse modelo na cidade de São Paulo: nessas UBS, 28,1\% trabalhavam há mais de 10 ou 20 anos, e apenas $16,8 \%$ há menos de 1 ano ou entre 1 e 2 anos. Nas UBS de Saúde da Família, 52,8\% dos entrevistados trabalhavam há menos de 1 ano ou entre 1 e 2 anos, o que se explica pela implantação do PSF no município ter se iniciado apenas em 2001, apesar do Qualis já existir desde 1996 na cidade. Houve uma expansão progressiva das equipes de Saúde da Família no município de São Paulo nos anos posteriores, embora em ritmo variável. Quanto a trabalhadores antigos, nessas UBS, 9,5\% informaram trabalhar na unidade há mais de 10 ou 20 anos: eram antigos funcionários de UBS, organizadas inicialmente sob o modelo dos Programas de Saúde, que a partir de 2001 foram reorganizadas sob o modelo de Saúde da Família. Os trabalhadores das UBS Mistas ocupavam uma posição "intermediária" quanto aos anos de inserção na UBS: $35 \%$ trabalhavam ali há mais de 10 ou 20 anos, e 34,6\% há menos de 1 ano ou até 2 anos.

\section{Experiência anterior de trabalho dos entrevistados}

Entre os entrevistados, 46,7\% apresentaram experiência anterior de trabalho na área hospitalar, sendo que destes 50,7\%, no momento da pesquisa, estavam trabalhando nas UBS de Programas de Saúde, 34,1\% nas UBS Mistas e 15,2\% nas UBS de Saúde da 
Família. Entre os que tiveram experiência anterior na área hospitalar, 19,3\% o fizeram apenas durante 1 ou 2 anos, $18,6 \%$ durante mais de 10 anos e outros $15,9 \%$ durante mais de 20 anos. Não foi possível apurar quantos haviam deixado o trabalho na área hospitalar e passaram a trabalhar exclusivamente na Atenção Básica, e quantos mantinham os dois tipos de trabalho.

\section{Contratos de trabalho}

Quase todos os entrevistados que trabalhava nas UBS organizadas sob o modelo de Programas de Saúde, isto é, 92,3\%, eram funcionários públicos estatutários e efetivos, e 4,7\% funcionários públicos regidos pela CLT. Os demais eram estatutários e recebiam complementação salarial pelas instituições parceiras da SMS-SP na implantação das Equipes de Saúde da Família, no regime CLT (tabela 3).

Tabela 3 - Distribuição do número e proporção (\%) dos Trabalhadores das UBS de Programas de Saúde, segundo Contrato de Trabalho, região Norte e STS Sé, São Paulo, 2009

\begin{tabular}{lcc} 
Forma de Contratação & $\mathrm{N}^{\circ}$ & $\%$ \\
\hline Duplo vínculo - Estatutário + CLT & 2 & 0,5 \\
Duplo vínculo - Estatutário + CLT Parceira & 2 & 0,5 \\
Outros & 6 & 1,6 \\
Parceira CLT & 1 & 0,3 \\
Público - Estatutário/Concursado/Efetivo/CLT & 336 & 92,3 \\
Público CLT & 17 & 4,7 \\
\hline Total & 364 & 100,0 \\
\hline
\end{tabular}

Fonte: Pesquisa ETAC, Cealag. São Paulo, 2009.

Entre os entrevistados que trabalhavam nas UBS de Saúde da Família ou entre os que trabalham nas equipes de Saúde da Família implantadas nas UBS Mistas a situação foi inversa: $77 \%$ apresentavam vínculo exclusivo pela CLT, contratados pelas Instituições Parceiras; 14,8\% eram funcionários públicos concursados; $6,6 \%$ apresentavam duplo vínculo (estatutários e CLT), recebendo complementação salarial através da Instituição Parceira da SMSSP, e 1,2\% eram funcionários públicos celetistas (tabela 4).
Tabela 4 - Distribuição do número e proporção (\%) dos Trabalhadores das Equipes de Saúde da Família por Tipo de Contrato, região norte e STS Sé, São Paulo, 2009

\begin{tabular}{lcc} 
Forma de Contratação no PSF & $\mathrm{N}^{\circ}$ & $\%$ \\
\hline Duplo vínculo - Estatutário + CLT & $\mathrm{I}$ & 0,4 \\
Duplo vínculo - Estatutário + CLT Parceira & 17 & 6,6 \\
Parceira CLT & 198 & 77,0 \\
Público - Estatutário/Concursado/Efetivo/CLT & 38 & 14,8 \\
Público CLT & 3 & 1,2 \\
\hline Total & 257 & 100,0 \\
\hline
\end{tabular}

Fonte: Pesquisa ETAC, Cealag. São Paulo, 2009

Número de serviços de saúde em que os entrevistados trabalhavam

No momento da pesquisa, $64,7 \%$ dos entrevistados trabalhavam só naquela UBS, enquanto outros $27,5 \%$ trabalhavam em dois serviços (incluindo essa UBS), $5,6 \%$ em três locais, $1,3 \%$ em quatro locais e $0,6 \%$ em mais de 4 locais. Apesar de muitos trabalharem em mais de um serviço de saúde, mais da metade trabalha em uma única UBS. Relacionando o número de serviços de saúde em que trabalhavam os entrevistados com os modelos de organização das UBS, constata-se que nos três tipos de UBS mais da metade dos entrevistados trabalhava apenas naquela UBS: nessa situação, encontravam-se $57,4 \%$ dos que trabalhavam nas UBS de Programas de Saúde, $67,3 \%$ dos que trabalhavam nas UBS Mistas e, $73,2 \%$ dos que trabalhavam nas UBS de Saúde da Família. O fato de ser proporcionalmente maior o número de trabalhadores que atuavam apenas um serviço nas UBS Mistas e UBS de Saúde da Família deve decorrer da exigência do trabalho em tempo integral para os componentes das Equipes de Saúde da Família.

A respeito dos grupos de entrevistados que informaram trabalhar em dois serviços: $31,3 \%$ deles trabalhavam nas UBS de Programas de Saúde, 25,5\% nas UBS Mistas e 24,2\% nas UBS de Saúde da Família. Em menor número, encontraram-se alguns que trabalhavam em três serviços e, dentre estes, 8,2\% trabalhavam em UBS de Programas de Saúde, 5,8\% em UBS Mistas e apenas 1,3\% nas UBS de Saúde da Família (gráfico 2). 


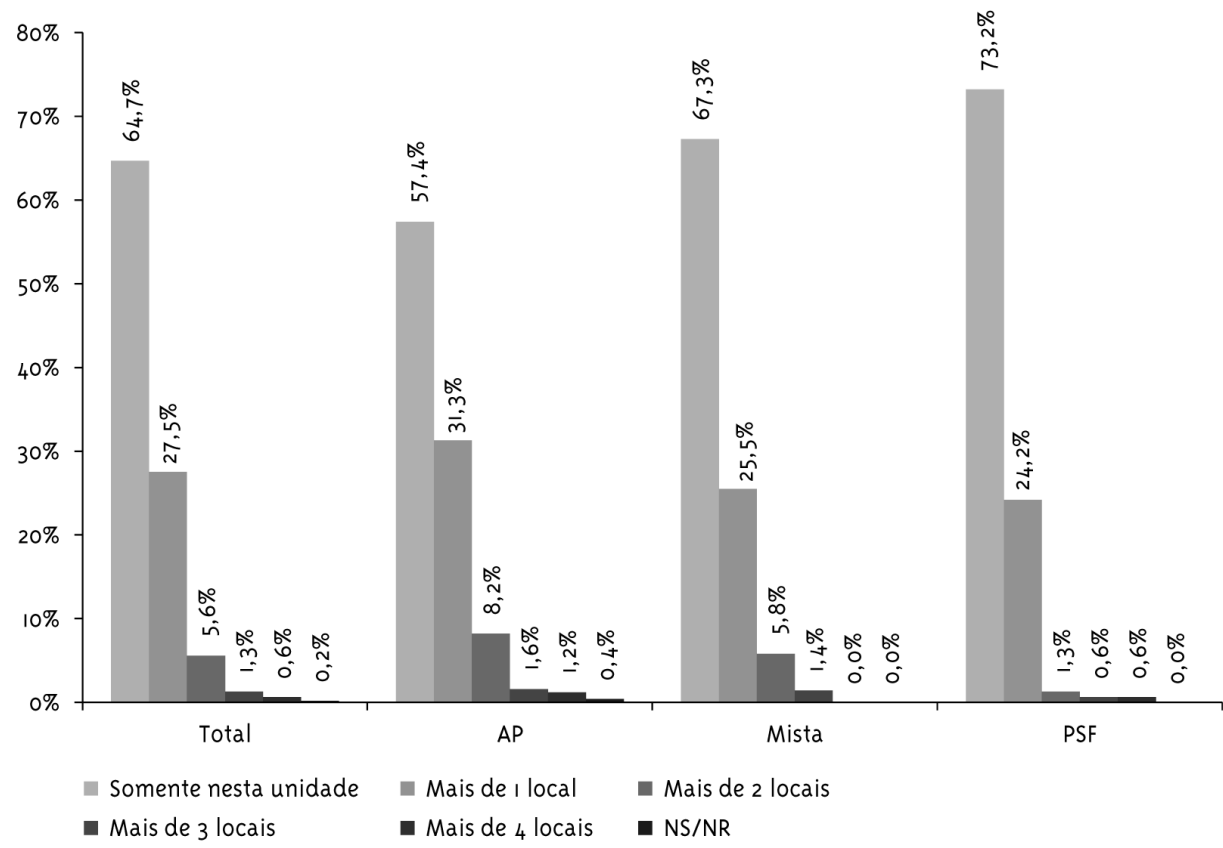

Fonte: Pesquisa ETAC, Cealag. São Paulo, 2009.

\section{Discussão e Considerações Finais}

Esta pesquisa confirma as observações de vários autores sobre complexidade, diversidade e especificidade da Atenção Básica nas grandes cidades e os desafios para a Estratégia de Saúde da Família nessas áreas: preexistência de uma rede de Atenção Básica desenvolvida anteriormente, mix público e privado no setor saúde, maior nível de escolaridade da população, especialmente feminina, espaços urbanos heterogêneos e desiguais socialmente (Vianna e Silva, 2005; Cohn e col., 2005).

Analisando-se o universo dos trabalhadores das 87 UBS existentes nas regiões pesquisadas, confirmou-se a tendência de feminização da força de trabalho na Atenção Básica, embora menor entre os médicos.

A existência de dois importantes contingentes de trabalhadores, um entre 21 e 40 anos e outro entre 41 e 60 anos, indica dois movimentos que se comple- mentam: de um lado, a antiguidade e a consolidação da inserção de um contingente de trabalhadores na Atenção Básica e, de outro, a renovação dos recursos humanos, o que se confirma pelo fato de $19,2 \%$ dos entrevistados trabalharem há mais de 10 ou 20 anos na mesma unidade, $20,9 \%$ há 5 ou 6 anos, e 37,9\% entre 1 e 4 anos.

A maioria dos entrevistados era natural da região sudeste, 61,4\% do município de São Paulo, apresentando escolaridade muitas vezes superior ao exigido para o exercício dos cargos e funções em que estão inseridos, tendo realizado vários Cursos de Espacialização na área de Saúde Pública ou Clínica Médica, de acordo com os Programas de Saúde em que estão inseridos, ou Cursos de Nível Técnico, especialmente de enfermagem ou da área de administração.

Entre os profissionais de nível universitário, o maior contingente era de médicos, seguido do de enfermeiros e cirurgiões-dentistas; outros profissionais foram encontrados em menor número: 
assistentes sociais e psicólogos e, em menor número ainda, apenas em parte das UBS, nutricionistas, fisioterapeutas, terapeutas ocupacionais e farmacêuticos.

Entre os trabalhadores de nível médio, predominaram os auxiliares e, entre eles, os auxiliares de enfermagem. Os trabalhadores de nível técnico compunham um pequeno número e a maioria deles atuava na área administrativa.

Entre os trabalhadores de nível operacional, os ACS formavam um maior número, como resultado da expansão das Equipes de Saúde da Família a partir de 2001 no município de São Paulo.

Quanto à experiência anterior de trabalho, 46,7\% informaram ter trabalhado na área hospitalar e $66,7 \%$, no momento da pesquisa, estavam trabalhando apenas naquela UBS.

As UBS da Supervisão Técnica de Saúde Sé contavam com maior número de trabalhadores do que as UBS da Região Norte, em que as UBS da Vila Maria/ Vila Guilherme e Santana/Jaçanã eram as que, em média, tinham o menor número de trabalhadores.

Considerando-se a distribuição das 87 UBS por modelo de organização, constatou-se que quase metade delas estava organizada exclusivamente segundo o modelo dos Programas de Saúde, enquanto $26,5 \%$ se transformaram exclusivamente no modelo de Saúde da Família, e 24,1\% se tornaram UBS Mistas.

O processo de feminização pareceu um pouco mais acentuado nas UBS de Saúde da Família, provavelmente pela incorporação dos ACS, quase totalmente pertencentes ao sexo feminino. Nesse tipo de UBS, havia também um contingente de trabalhadores ligeiramente mais jovem do que nas UBS organizadas sob os outros modelos.

Quanto à distribuição dos trabalhadores, embora no universo o número de médicos tenha sido praticamente o dobro do de enfermeiros, nas UBS de Saúde da Família, o número de enfermeiros foi ligeiramente superior ao de médicos, em virtude da composição das equipes.

Considerando-se a distribuição do contingente de trabalhadores, segundo o nível de cargos ocupados e o modelo de organização das UBS, constatou-se que: a) nas UBS do modelo de Programas de Saúde, havia proporcionalmente mais trabalhadores de nível médio (auxiliares e técnicos), seguidos de profissionais de nível superior e um número bem menor de operacionais; b) nas UBS de Saúde da Família, o maior número foi de trabalhadores de nível operacional (aqui é preciso considerar o peso dos ACS), seguidos de auxiliares e técnicos e, um menor número, profissionais de nível universitário; c) nas UBS Mistas, havia um maior contingente de auxiliares e técnicos, seguido de trabalhadores de nível operacional (os ACS da equipes de Saúde da Família), e um menor número de profissionais de nível superior.

Nas UBS de Programas de Saúde, o contingente de trabalhadores com mais de 40 anos de idade e com mais de 10 anos de trabalho na mesma UBS era maior, enquanto nas UBS de Saúde da Família, havia um contingente maior de trabalhadores com menos de 40 anos de idade e menor tempo de inserção na UBS, muitos abaixo de 5 anos, e outros entre 1 e 2 anos.

Experiência anterior na área hospitalar foi relatada por um maior número de trabalhadores das UBS organizadas sob os Programas de Saúde do que pelos que trabalhavam nas UBS organizadas exclusivamente sob o modelo de Saúde da Família. 0 número de trabalhadores que referiu trabalhar apenas naquela UBS em que estava no momento da pesquisa, e não ter outros vínculos de trabalho, foi maior entre os das UBS de Saúde da Família.

Quanto ao tipo de vínculo de trabalho, nas UBS de Programas de Saúde a quase totalidade (92,3\%) era composta de funcionários estatutários, enquanto nas UBS de Saúde da Família, 77\% dos entrevistados eram contratados por uma das instituições parceiras da SMS-SP, via CLT, e 14,8\% eram funcionários estatutários. Constata-se, assim, que na cidade de São Paulo, na implantação da Estratégia de Saúde da Família, a administração municipal de saúde optou pela terceirização da força de trabalho, não se observando, nas UBS estudadas, a precarização das relações de trabalho, como têm sido apontada por vários autores ao analisar a questão no país, dentre eles, Nogueira (2002) e Medeiros e colaboradores (2007). 


\section{Referências}

BRASIL. Ministério da Saúde. Saúde da Família: uma estratégia para a reorientação do modelo assistencial. Brasília: Ministério da Saúde, 1997.

BRASIL. Ministério da Saúde. Ministério da Educação. Portaria Interministerial n. 2101, de o3 de novembro de 2005. Institui o Programa Nacional de Reorientação da Formação Profissional em Saúde - Pró-Saúde - para os cursos de graduação em Medicina, Enfermagem e Odontologia. Brasília: Ministério da Saude; Ministério da Educação, 2005.

BRASIL. Ministério da Saúde. Ministério da Educação. Portaria Interministerial n.1802, de 26 de agosto de 20o8. Institui o Programa de Educação pelo Trabalho para a Saúde - PET Saúde. Brasília: Ministério da Saúde, Ministério da Educação, 2008.

COHN, A.; NAKAMURA, E.; COHN, C. O Programa de Saúde da Família entre o público e o privado. In: VIANNA, A. L. A.; ELIAS, P. E. M.; IBAÑEZ, N. (Orgs.) Proteção social: dilemas e desafios. São Paulo: HUCITEC, 2005. p. 169-1185.

JATENE, A. D. et al. $1^{\circ}$ Relatório Técnico-Científico: novos modelos de Assistência à Saúde: avaliação do Programa de Saúde da Família no Município de São Paulo. São Paulo: FAPESP, 2000.

LUPPI, C. G. et al. Integralidade, modelos organizacionais na Atenção Básica, avaliação: aspectos conceituais e conselhos operacionais. In: MARSIGLIA, R. M. G. (Coord.). Integridade e Atenção Primária em saúde: avaliação da organização do processo de trabalho em Unidades de Saúde da Secretaria Municipal da Saúde de São Paulo. São Paulo: CNPq, 20o8. Disponível em: <http://www.cealag. com.br/Trabalhos/INTEGRALIDADE\%20 E\%20ATEN\%C3\%87\%C3\%830\%20 PRIM\%C3\%81RIA\%2oEM\%20SA\%C3\%9ADE/ Relat\%C3\%B3rio.pdf>. Acesso em: 21 maio 2010.

Recebido em: 18/05/2011

Aprovado em: 03/07/2011
MARSIGLIA, R. M. G. Servidor, funcionário, trabalhador de saúde: organização de interesses e culturas organizacionais no setor público de saúde. 1993. Tese (Doutorado em Ciência Política) - Faculdade de Filosofia, Letras e Ciências Humanas, Universidade de São Paulo, São Paulo, 1993.

MARSIGLIA, R. M. G.; CARNEIRO JUNIOR, N. O PSF e os princípios do SUS: disponibilidade, acessibilidade e aceitabilidade do PSF em áreas metropolitanas. In: COHN, A. (Org.). Saúde da Família e SUS: convergências e dissonâncias. Rio de janeiro: Beco do Azougue; São Paulo: CEDEC, 2009. p. 93-112.

MARSIGLIA, R. M. G.; IBAÑEZ, N.; IANNI, A. M. Z. A experiência do Pró-Saúde na Faculdade de Ciências Médicas da Santa Casa de São Paulo. In: PIERANTONI, C. R.; VIANNA, A. L. A. (Orgs.). Educação \& Saúde. São Paulo: HUCITEC, 2010. p. 49-8o.

MEDEIROS, S. M. et al. Condições de trabalho, riscos ocupacionais e trabalho precarizado: o olhar dos trabalhadores de enfermagem. In: CASTRO, J. L. (Org.). Gestão do trabalho no SUS: entre o visível e o oculto. Natal: Editora Observatório RH/NESC/UFRN, 2007. p. 161-200.

NEMES, M. I. B. Ação programática em saúde: recuperação histórica de uma política de programação. In: SCHRAIBER, L. B. (Org.). Programação em saúde hoje. São Paulo: HUCITEC, 1993. p. 65-116.

NOGUEIRA, R. P. O trabalho em saúde hoje: novas formas de organização. In: NEGRI, B.; FARIA, R.; VIANNA, A. L. A. (Org.). Recursos humanos em saúde: política, desenvolvimento e mercado de trabalho. Campinas: Unicamp, 2002. p. 257-273.

SILVA, J. A.; DALMASO, A. S. W. Agente Comunitário de Saúde: o ser, o saber, o fazer. Rio de Janeiro: Fiocruz, 2002.

VIANNA, A. L. A.; SILVA, H. P. Saúde em grandes centros. In: VIANNA, A. L. A.; ELIAS, P. E. M.; IBAÑEZ, N. (Orgs.) Proteção social: dilemas e desafios. São Paulo: HUCITEC, 2005. p. 189-210. 